\title{
Assessment of Serum Vitamin D Levels in Women with Polycystic Ovary Syndrome
}

\section{Osama Mansour Khalifa ${ }^{1, *}$ MSc., Esmael Mohammed El garhy ${ }^{2}$ PhD., Fahd Abdelaal Elomda ${ }^{2}$ PhD. and Mohammed Basiouny Yehia ${ }^{2}$ MD.}

\section{* Corresponding Author: \\ Osama Mansour Khalifa \\ osamamansour1985@gmail.com}

Received for publication September 21, 2021; Accepted November 19, 2021; Published online November 19, 2021.

Copyright The Authors published by Al-Azhar University, Faculty of Medicine, Cairo, Egypt. Users have the right to read, download, copy, distribute, print, search, or link to the full texts of articles under the following conditions: Creative Commons Attribution-Share Alike 4.0 International Public License (CC $B Y-S A$ 4.0).

doi: 10.21608/aimj.2021.96806.1582

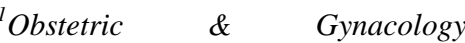
Department, Egyptian Military Medical Academy, Cairo, Egypt.

${ }^{2}$ Obstetric \& Gynacology Department, Faculty of Medicine, Al-Azhar University Cairo, Egypt.

\begin{abstract}
Background: Polycystic ovary syndrome is the most common endocrine problems between femaleof the reproductive age and represent single most endocrine-metabolic problem in female. Its incidence has been estimated globally and in different countries around $5-10 \%$.

Vitamin D, is considered a fat soluble vitamin, there are 2 ways for synthesis of Vitamin D.The first way is through the intestinal absorption and internal production from a precursor of 17-hydroxyl cholesterol on the skin with good exposure to ultraviolet sunlight. Vitamin D level less than $20 \mathrm{ng} / \mathrm{ml}$ is deficiency.

Aim of the work: to assess the level of vitamin D in female have polycystic ovary syndrome.

Patients and methods: This study is case control study it was done at Misr Al-Gadeda Military Hospital outpatient clinic from May 2020 May 2021.Patients recruited from attendees of the outpatient gynecological clinic and inpatient ward at Misr Al-Gadeda Military Hospital.

Group A: 45 women diagnosed with PCO based on Rotterdam Criteria2003.

Group B: 45 healthy women are selected from outpatient clinic.

Results: The results showed that $25(\mathrm{OH})$ vitamin D levels were remarkably decreased in PCOS group in comparison to healthy women. There were no obvious relations between vitamin D levels, age and body mass index.

Conclusion: Twenty five $(\mathrm{OH})$ Vitamin D was significantly lower in women with PCOS have low level of vitamin D than healthy women. No remarkable changes in Vitamin D levels related to age group and body mass index.
\end{abstract}

Keywords: Vitamin D level; Assessment; PCOS.

Disclosure: The authors have no financial interest to declare in relation to the content of this article. The Article Processing Charge was paid for by the authors.

Authorship: All authors have a substantial contribution to the article.

\section{INTRODUCTION}

Women at reproductive age are facing many problems which annoying their life and affects their physical activities, one of this problem and consider to be famous problem are Polycystic ovary syndrome (PCOS).It is endocrine problem and represent single most endocrine metabolic problem in female. Many studies and researches have reported that PCOS incidence in different countries is nearly $5-10 \%$. These women are more exposed to have menstrual irregularities, hyper androgenism, and an ovulatory cycles and polycystic ovaries. ${ }^{1}$

There are many studies which tried to estimate incidence of polycystic ovary syndrome in Egypt, approximately it is $13 \%$ in female have birth and $37.5 \%$ in patients complaining of infertility either primary or secondary. ${ }^{2}$

It can produce chronic problems as DM, endocrine gland cancer and cardiovascular diseases it is associated with anovulation, increased insulin secretion, and central obesity.
PCOS is mainly accompanied with metabolic problems, including increased weight, disturbance of glucose metabolism, and insulin resistance (IR). ${ }^{4}$

PCOS is considered to be one of the common factors causing an ovulatory infertility. ${ }^{5}$

Many features for diagnosis used for PCOS diagnosis according to these Rotterdam criteria, the assessment can be established with the finding of at least 2 of 3 of these criteria: anovulation, clinical symptoms and laboratory findings of excess androgen or the finding of PCOS picture has been confirmed by ultrasonography. ${ }^{6}$

Impaired glucose metabolism, increased Resistance to insulin, increased body weight, hyperlipidemia and hypertension are the essential picture of metabolic syndrome. Resistance to insulin has a vital role in the development of polycystic ovary syndrome.

Approximately it is estimated that $50-80 \%$ of polycystic ovary syndrome patients complaining from IR. ${ }^{8}$ 
In recent studies it has been shown that vitamin $\mathrm{D}$ deficiency (VDD) is associated with resistance to insulin, and this can lead to influence on the amount of insulin secreted by the beat cell of pancreas.'

Results of a lot of studies had proved important evidence that link between overweight with VDD. This gives chance to ask about the relationship of overweight and VDD in the development of polycystic ovary syndrome between women at risk to develop this syndrome. This research was done to discover the role of serum vitamin D levels on insulin resistance, metabolic parameters in patients have normal weight and diagnosed with polycystic ovary syndrome. ${ }^{10}$

Vitamin D is considered a fat soluble vitamin, there are 2 ways for production of Vitamin $D$, and the first way is through the intestinal absorption and internal production from a precursor of 17-hydroxyl cholesterol on the skin with good exposure to ultraviolet sunlight. Vitamin is considered to be deficient if it is less than $20 \mathrm{ng} / \mathrm{ml}$ and this is reported by most of the authors. ${ }^{11}$

There is association between vitamin D levels, overweight, other metabolic items in polycystic ovary syndrome women are reported by most of previous studies this metabolic parameters include the fasting Insulin, Fasting glucose levels, IR, hypertension, dyslipidemia, fertility and other clinical and laboratory-related parameters associated with polycystic ovary syndrome. ${ }^{12}$

\section{PATIENTS AND METHODS}

The current study held in Misr Al-Gadeda Military Hospital in outpatient clinic, from May 2020 to May 2021 and it is a case control study.

Group A: 45 women with PCOS based on Rotterdam Criteria 2003 which defined the presence of two of the following three characters at least: oligoovulation, increased androgen levels (clinical, laboratory), morphological features of polycystic ovary detected by ultrasound.

Group B: 45 fertile women without polycystic ovary syndrome who are recruited from the outpatient gynecological clinic and inpatient gynecological ward.

\section{Inclusion criteria}

Age 18-35.

Patients with PCOS according to Rotterdam Criteria 2003 which referred as the presence of at least two of the following three standard criteria:

Chronic oligo/amenorrhea and/or chronic anovulation.

Clinical and/or biochemical signs of hyperandrogenism.

Morphological features of polycystic ovaries discovered by ultrasound (i.e., having ovarian volume of over $10 \mathrm{~cm} 3$ and/or presence of more than 12 ovarian follicles of $2-9 \mathrm{~mm}$ in length).

\section{Exclusion criteria}

Women with endocrine problems as(thyroid, parathyroid, DM, Cushing syndrome, CAH, increased prolactin level.

Women have renal, liver or chronic disease.

History of using vitamin D supplements or any medication affecting vitamin D level within 6 months before the study.

Women have smoking history, drug abuse.

Women have a history of hormonal treatment in the last 3 months prior to the study.

Female patient complaining of fibroid uterus

\section{All patients subjected to the following:}

Detailed History: including the following points.

Personal history (name, age, residence, occupation, special habits).

Present history.

Surgical history (previous operation).

Women who have the history of recent hormonal contraception and especial drug intake.

History of a similar condition in the family.

Menstrual history of menarche, LMP and the amount of bleeding.

\section{Examination}

\section{General examination}

This includes the weight, height, BMI $(\mathrm{kg} / \mathrm{m} 2)$

General characteristics for hirsutism

Vital signs which includes the following (blood pressure and pulse), pallor,signs of anemia.

Local examination

Examinations external genitalia of

Ultrasound

For case and control groups.

Laboratory Investigations Serum vitamin D level.

Complete blood picture.

Hormonal profile (T3, T4, TSH, Prolactin,

$\mathrm{FSH}$, and LH).

\section{INTERVENTION:}

PCOS women and controls have the following:

Weight was measured in light indoor clothing and barefoot and height was measured in stand up position. Then, the following equation was used to calculate BMI.

$\mathrm{BMI}=\mathrm{kg} / \mathrm{m}^{2}$.

According to BMI findings, the participants were

divided into four following groups:

Less than $18.5 \mathrm{~kg} / \mathrm{m}^{2}$ as underweight.

$18.5-22,9 \mathrm{~kg} / \mathrm{m} 2$ as normal weight.

$23-24.9 \mathrm{~kg} / \mathrm{m} 2$ as overweight.

$25 \mathrm{~kg} / \mathrm{m} 2$ or more as obese. ${ }^{14}$

2- Vitamin Dlevels were assessed using Chemilumiscence ImmuneAssay (CLA)on LIAISON Device. Heamolized sample was rejected.

Technique for serum vitamin D measurement Specimen collection and handling

The blood sample was collected by puncture of vein. The sample was kept in standard sampling tube without anticoagulant, clotting was allowed. Then the tube sample was centrifugated at $4000 \mathrm{rpm}$. This 
results in plasma separation from other components. Vitamin D level in plasma were analysed using Chemilumencent Immune Assay by LIAISON device (DIASORIN), the used kits was (Ref 310600) manufactured by DAISORIN company, USA.

\section{Principle of the test}

LIAISON laboratory Diagnostic Vitamin D total assessment using a competitive chemiluminscent protein binding assessment technology for the quantitative determination of $25(\mathrm{OH})$ vitamin $\mathrm{D}$ and other hydroxylated vitamin D in human serum EDTA -plasma or Lithium heparin plasma to be used in vitamin D deficiency assessment using the Liaison family analyzer.

LIAISON is modified in 3 steps sandwich assessment process that use recombinant protein fusion for capture of $1,25(\mathrm{OH}) \mathrm{D}$ molecule and monoclonal antibody which specifically recognizes the complex formed by the recombinant fusion protein with 1,25(OH) D Molecule. Result should be used in conjugation with other clinical and laboratory data to help the physician in making individual decision for every patient regarding management.

The assay use 3 steps incubation process with overall duration of 65 minutes.

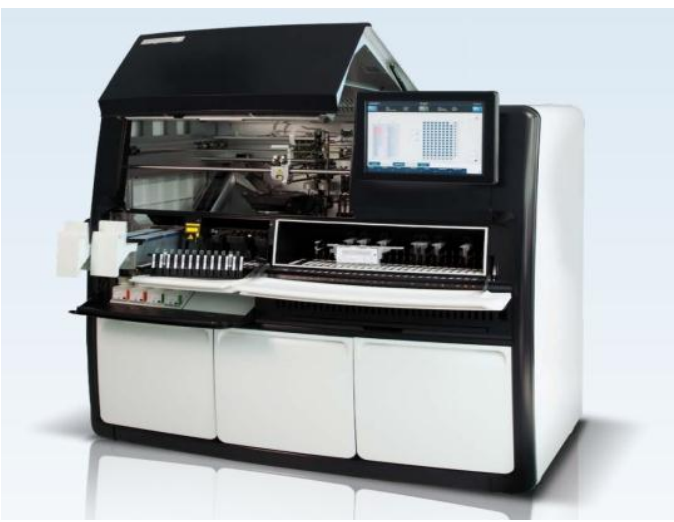

Fig. 1: LIAISON device.

\begin{tabular}{|l|l|}
\hline Deficient & $<20 \mathrm{ng} / \mathrm{ml}$ \\
\hline insufficiency & $20 \mathrm{ng} / \mathrm{ml}-30 \mathrm{ng} / \mathrm{ml}$ \\
\hline sufficient & $30 \mathrm{ng} / \mathrm{ml}-100 \mathrm{ng} / \mathrm{ml}$ \\
\hline intoxication & $>100 \mathrm{ng} / \mathrm{ml}$ \\
\hline
\end{tabular}

Table 1: Reference range for vitamin D level. ${ }^{1}$

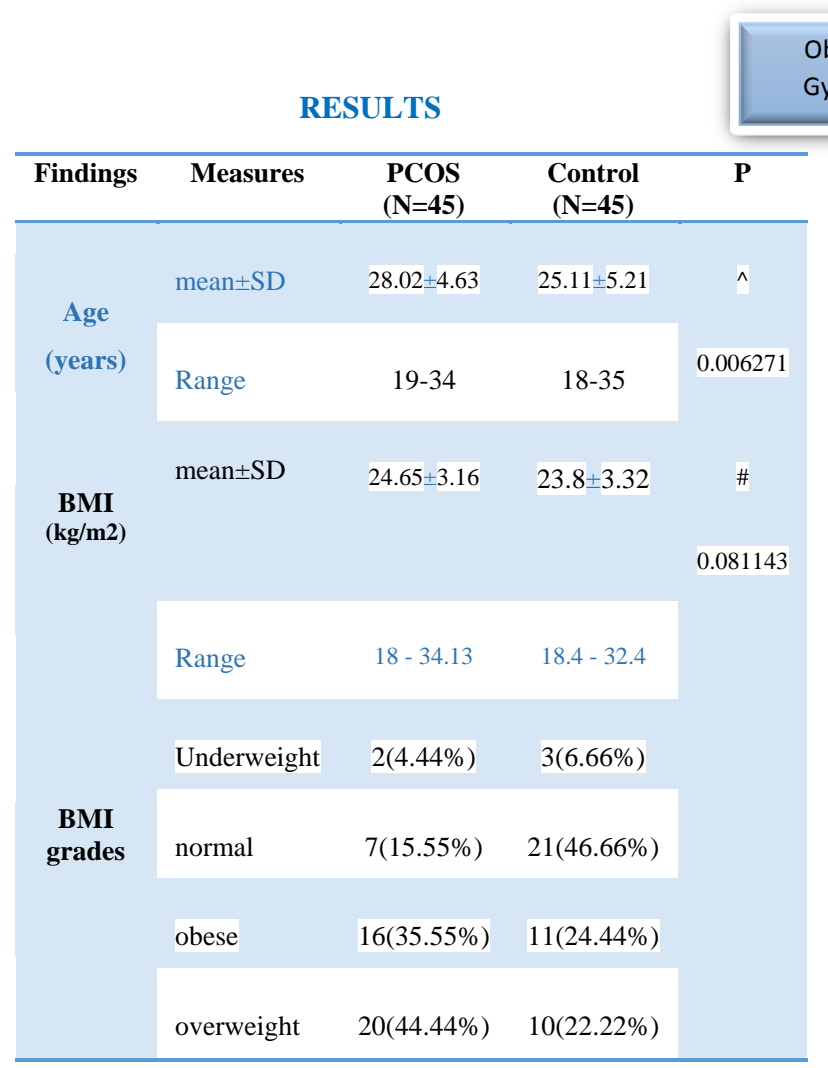

${ }^{\wedge}$ Independent t-test, \#Fisher's Exact test, *Significant

Table 2: Comparison between the studied groups regarding age and BMI

\begin{tabular}{cccc}
\hline & \multicolumn{2}{c}{$\begin{array}{c}\text { VIT D } \\
\text { level(ng/ml) }\end{array}$} & \multirow{2}{*}{ P* $^{*}$} \\
\cline { 2 - 3 } & Mean & SD & \\
PCOS & 15.46 & 9.48 & \\
CONTROL & 22.4 & 11.35 & 0.01268 \\
\hline
\end{tabular}

Table 3: Vitamin D levels in women with PCOS andhealthy women

\begin{tabular}{ccccc}
\hline Findings & Measures & $\begin{array}{c}\text { PCOS } \\
(\mathbf{N}=45)\end{array}$ & $\begin{array}{c}\text { Control } \\
(\mathbf{N}=45)\end{array}$ & P \\
\hline & mean \pm SD & $15.64 \pm 9.48$ & $22.4 \pm 11.35$ & $\hat{0}$ \\
level(ng/ml) & Range & $3-34$ & $5-38$ & \\
& Normal & $5(11.1 \%)$ & $18(40 \%)$ & \\
Grades & insufficiency & $10(22.2 \%)$ & $17(37.7 .6 \%)$ & $\begin{array}{c}\# \\
0.00032\end{array}$ \\
& Deficiency & $25(55.5 \%)$ & $10(22.2 \%)$ & \\
\hline
\end{tabular}

${ }^{\wedge}$ Independent t-test, \#Fisher's exact test significant

Table 4: comparison between the studied groups regarding $25(\mathrm{OH})$ vitaminD level. 


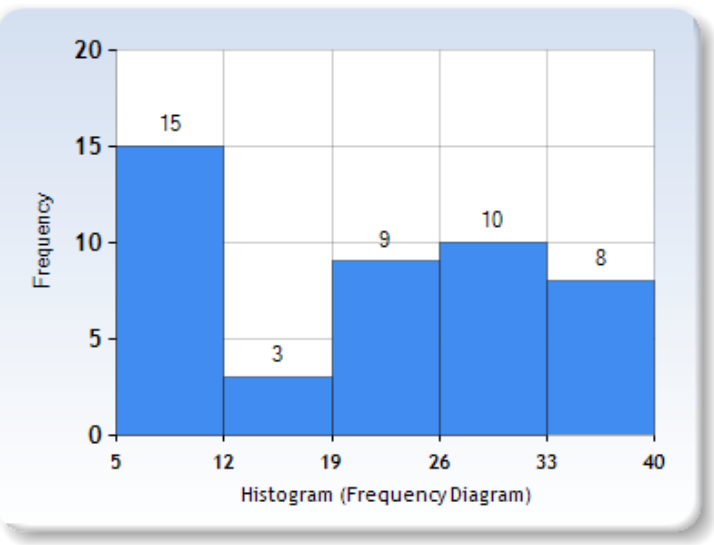

Fig. 2: Histogram showing Vitamin D level among control group

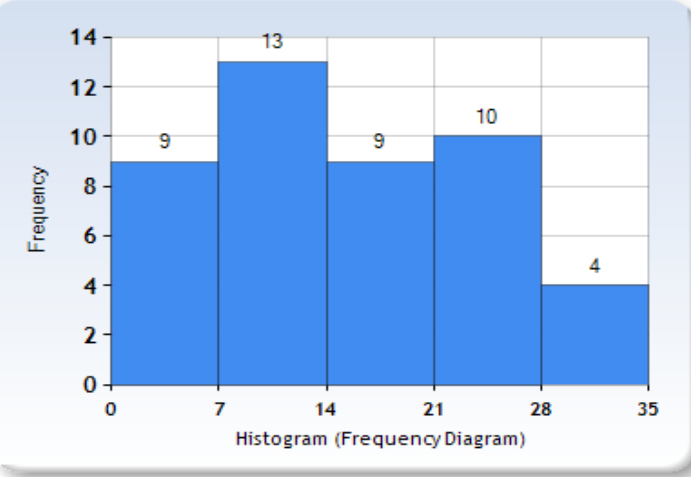

Fig. 3: Histogram for Vitamin D level among PCOS groups.

\begin{tabular}{cccc}
\hline $\begin{array}{c}\text { Vitamin D } \\
\text { deficiency }\end{array}$ & Case & Control & Total \\
\hline$<\mathbf{1 0}$ & $19(59.37)$ & $13(40.62)$ & 32 \\
\hline $\mathbf{1 0}>-\mathbf{2 0}$ & $12(75 \%)$ & $4(25 \%)$ & 16 \\
\hline Total & 31 & 17 & 48 \\
\hline
\end{tabular}

Table 5: Vitamin D deficiency among PCOS and control group.

\begin{tabular}{lc}
\hline Number of Cases & $\mathbf{9 0}$ \\
\hline Number Correct & 58 \\
\hline Accuracy & $64.4 \%$ \\
Sensitivity & $88.9 \%$ \\
\hline Specificity & $40.0 \%$ \\
\hline Positive Cases Missed & 5 \\
\hline Negative Cases Missed & 27 \\
\hline Fitted ROC Area & 0.62 \\
Empiric ROC Area & 0.627 \\
\hline Positive predictive value & $63.9 \%$ \\
\hline Negative predictive value & $81.8 \%$ \\
Positive like hood ratio & 1.48 \\
\hline Negative like hood ratio & 0.28 \\
\hline
\end{tabular}

Table 6: vitamin D levels diagnostic characteristics in diagnosis of PCOS.

\section{DISCUSSION}

Many people in different age groups are complaining of Vitamin D Deficiency, vitamin D deficiency is considered worldwide problem. ${ }^{14}$

In the last few years many studies and researches try to discover the correlation between vitamin $\mathrm{D}$ levels and PCOS, it is reported that women complaining of PCOS have high chance of vitamin D deficiency than healthy women. ${ }^{15}$

In agreement with us Ellida et al., have study which agrees with our study, his study found that levels of vitamin $\mathrm{D}$ are higher in control groups in comparison to Polycystic Ovarian Syndrome women. 46 women were assessed in regard to the following it which was the age and the Body Mass Index. Vitamin D levels negative relation with Body Mass Index. Obese women have a very low level vitamin D. This difference was statistically significant.

There is a case control study on 38 women with PCOS, 109 women were control and the study make comparison for vitamin D levels in women with PCOS with matched healthy group. Results indicate that PCOS groups have low level of vitamin D in comparison to healthy women. There were no remarkable relations between vitamin $\mathrm{D}$ in relation to age and body mass index. There is remarkable difference in vitamin D levels between PCOS women and healthy women.$^{16}$

In agreement with us $\mathrm{He} C \mathrm{C}$ et al. has confirmed that Polycystic Ovarian Syndrome women associated with vitamin $\mathrm{D}$ deficiency, the study confirm that approximately $67-85 \%$ of PCOS women have vitamin $D$ level below $20 \mathrm{ng} / \mathrm{mL} .^{17}$

Also study held of 80 newly diagnosed PCOS women with mean age (23 to 33 years). Many Items were assessed in this study which includes the following items FBS, Calcium, phosphorus, vitamin D. Results of PCOS and matched healthy Women were compared. Women with PCOS have low level of vitamin $\mathrm{D}$ in comparison to the healthy controls and there obvious difference with age and BMI . ${ }^{18}$

A study of 242 women, 125 PCOS women and 117 women were normal. The results indicated that both PCOS and control groups have higher levels of Vitamin D deficiency. Calcium and Vitamin D were decreased in PCOS women, vitamin D level was approximately the same among obese and normal weight women. ${ }^{19}$

This study carried on 314 infertile women. In the study 157 infertile women with PCOS case group, 157 healthy women. Mean serum vitamin D levels in PCOS women were significantly lower when compared to healthy women $(13 \pm 11.5 \mathrm{ng} / \mathrm{ml}$ and $25.07 \pm 20.7 \mathrm{ng} / \mathrm{ml})$. Infertile PCOS women were more prevalent to vitamin $\mathrm{D}$ deficiency than the other infertile patients. Therefore, measuring serum vitamin D especially in infertile PCOS was suggested. ${ }^{20}$ 
While study carried on 99 women with PCOS and66 healthy women. Their result indicates that there was remarkable difference in vitamin D levels among women with PCOS and also in the prevalence of VDD. PCOS women with vitamin D deficiency had obviously BMI with higher level and increased level of fasting insulin in comparison to Vitamin D Deficient controls. $^{21}$

Study on60 PCOS women and 90 healthy women. The study concluded women with PCOS have serum vitamin $\mathrm{D}$ levels of low level in comparison to healthy women but this difference was not remarkable. The findings from all women in the study concluded that, out 150 women, 76 with vitamin D deficiency, 69 with vitamin D insufficiency and 5 women with a sufficient level. Between the PCOS subjects, 39 women had a deficient level and 21 had an insufficient level. Among the healthy women, there were 37 women with a deficiency, 48 with an insufficiency and 5 with a sufficient level. ${ }^{22}$

Also another study Of 639 women with PCOS and 449 healthy women. Serum vitamin D levels was remarkably decreased in women with PCOS in comparison to healthy women. ${ }^{23}$

A study of 40 patients with PCOS and 40 patient controls. Women with PCOS have low level of vitamin $D$ level than the healthy women.$^{24}$

In agreement with us a study on 169PCOS women and114 controls women. Vitamin D levels and metabolic markers were evaluated. The primary results was the different level of vitamin $\mathrm{D}$ between PCOS and healthy women, the secondary results was the relation between metabolic problems and vitamin $\mathrm{D}$ levels in women with PCOS. ${ }^{25}$

\section{CONCLUSION}

Females during the age of reproduction are facing many endocrine problems and PCOS is considered the commonest one.

Women having PCOS showed decreased levels of Vitamin D in comparison to healthy women

Unremarkable difference for Vitamin D level was discovered relation to age group and body mass index between PCOS and control groups.

\section{REFERENCES}

1. Susan M Sirmans and Kristen A Pate: Epidemiology, diagnosis, and management of polycystic ovary syndrome. ClinEpidemiol. 2014; 6: 1-13.

2. Sanad AS. Prevalence of polycystic ovary syndrome among fertile and infertile women in Minia Governorate, Egypt. Int $J$ Gynaecol Obstet.2014Apr; 125(1):812.

3. Haoula Z, Salman M and Atiomo W. Evaluating the association between endometrial cancer and polycystic ovary syndrome. Hum Reprod. 2012; 27(5):132731.

4. Couto Alves A, Valcarcel B, Mäkinen VP, et al. Metabolic profiling of polycystic ovary syndrome reveals interactions with abdominal obesity. Int $J$ Obes (Lond). 2017; 41(9):133140.

5. El Hayek S, Bitar L, Hamdar LH, et al. Poly Cystic Ovarian Syndrome: An Updated Overview. Front Physiol. 2016; 7:124.

6. Rotterdam ESHRE/ASRM-Sponsored PCOS consensus workshop group. Revised 2003 consensus on diagnostic criteria and long-term health risks related to polycystic ovary syndrome (PCOS). Hum Reprod. 2004; 19(1):41-7.

7. Amar Nagesh Kumar, JupalleNagaiah Naidu, UppalaSatyanarayana, et al. Association between insulin resistance, $25(\mathrm{OH})$ vitamin D levels in Indian women with PCOS International Journal of Clinical Biochemistry and Research.2015; (2):22-6.

8. DeUgarte CM, Bartolucci AA and Azziz R. Prevalence of insulin resistance in the polycystic ovary syndrome using the homeostasis model assessment. FertilSteril. 2005; 83(5):1454-60.

9. Garg G, Kachhawa G, Ramot R, et al. Effect of vitamin $\mathrm{D}$ supplementation on insulin kinetics and cardiovascular risk factors in polycystic ovarian syndrome: a pilot study. Endocr Connect. 2015; 4(2):108-16.

10. Pereira-Santos M, Costa PR, Assis AM, et al. Obesity and vitamin D deficiency: a systematic review and meta-analysis. Obes Rev. 2015; 16(4):341-9.

11. Holick MF and Chen TC: Vitamin D deficiency. New England journal of medicine.2012; 357 (3), 266-81.

12. Lerchbaum $\mathrm{E}$ and Obermayer-Pietsch B. Vitamin D and fertility: a systematic review. Eur J Endocrinol. 2012; 166(5):765-78.

13. ElidaSidabutar, Binarwan Halim, Muhammad Fidel GanisSiregar, et al. Vitamin D levels in women with polycystic ovary syndrome. ASPIRE Conference Proceedings, The 6th Congress of the Asia Pacific Initiative on Reproduction. KNE Medicine.2016; 125:88-95.

14. Holick MF. The vitamin D deficiency pandemic: Approaches for diagnosis, treatment and prevention. Rev EndocrMetabDisord. 2017; 18(2):153-65. 
15. Thomson RL, Spedding $S$ and Buckley JD. Vitamin D in the aetiology and management of polycystic ovary syndrome. Clin Endocrinol (Oxf). 2012; 77(3):343-50.

16. Kim J, Choi $\mathrm{Y}$, Chae $\mathrm{S}$ et al. vitamin $\mathrm{D}$ deficiency in women with polycystic ovary syndrome. ClinExp Reprod Med. 2014; 41(2): 80-5.

17. He C, Lin Z, Robb SW, et al. Serum Vitamin D Levels and Polycystic Ovary syndrome: A Systematic Review and Meta-Analysis. Nutrients. 2015; 7(6):4555-77.

18. Moini A, Shirzad N, Ahmad M, et al. Comparison of 25 hydroxyvitamin $\mathrm{D}$ and calcium levels between polycystic ovarian syndrome and normal women. International Journal of Fertility and Sterility.2015; 9:1-8

19. Maghbooli Z, Hossein-Nezhad A, Karimi F, et al. Correlation between vitamin D3 deficiency and insulin resistance in pregnancy. Diabetes Metab Res Rev. 2008; 24(1):27-32.

20. Alzoubi A, Mahdi H, Al Bashir S, et al. Normalization of serum vitamin $d$ improves semen motility parameters in patients with idiopathic male infertility. ActaEndocrinol. 2017; 13(2):180-7.
21. Figurová J, Dravecká I, Javorský $M$, et al.. Prevalence of vitamin D deficiency in Slovak women with polycystic ovary syndrome and its relation to metabolic and reproductive abnormalities. Wien KlinWochenschr. 2016; 128(17-18):641-8.

22. Krul-Poel YHM, Koenders PP, SteegersTheunissen RP, et al. Vitamin D and metabolic disturbances in polycystic ovary syndrome (PCOS): A cross-sectional study. PLos One. 2018; 13(12):e0204748.

23. Rahsepar M, Mahjoub S, Esmaelzadeh $\mathrm{S}$, et al. Evaluation of vitamin D status and its correlation with oxidative stress markers in women with polycystic ovary syndrome. Int $J$ Reprod Biomed. 2017; 15(6):345-50.

24. Sanaa ElKholy, Rowaa ElKholy, Amr Riyadh, et al. Assessment of vitamin D levels in women with polycystic ovarian syndrome. Egyptian Journal of Hospital Medicine. 2018; 70(4): 594-600

25. Wang L, Lv S, Li F, et al. Vitamin D Deficiency Is Associated With Metabolic Risk Factors in Women With Polycystic Ovary Syndrome: A Cross-Sectional Study in Shaanxi China. Front Endocrinol (Lausanne). 2020; 11:171. 